\title{
Coronal views of the paediatric mandibular condyle on computerised tomography brain (CTB) studies: An important review area
}

\section{T Peedikayil, $\mathbf{N}$ Wieselthaler}

Department of Radiology, Groote Schuur Hospital, Cape Town

T Peedikayil, $\mathrm{MB} \mathrm{ChB}$

Department of Radiology, Red Cross War Memorial Children's Hospital, Cape Town

N Wieselthaler, MB ChB, FCRad (Diag) SA

Corresponding author: T Peedikayil (mustardcp@yahoo.com)

\begin{abstract}
This article highlights the importance of routinely reviewing the mandibular condyle on computerised tomography brain studies in a trauma setting, that often extends to involve the mandibular condyles in the scan parameters. We discuss 4 cases seen over the last 18 months at our institution, and review the relevant literature regarding paediatric mandibular fractures.
\end{abstract}

S Afr J Rad 2013;17(1):38-39. DOI:10.7196/SAJR.779

We discuss 4 cases seen over the last 18 months at Red Cross War Memorial Children's Hospital in an effort to highlight the importance of routinely reviewing coronal views of the mandibular condyle when interpreting trauma-related computerised tomography brain (CTB) studies in a paediatric setting.

The mandible is the second most commonly fractured facial bone in children, after nasal bone fractures. ${ }^{[1,2]}$ The condyle is the most frequently fractured region of the mandible owing to its prominent vascularisation and medullary bone and relatively thin surrounding cortical bone. ${ }^{[3]}$

The 4 patients who presented to the trauma unit varied in age from 2 - 12 years. Two of them presented after a motor vehicle accident (MVA), 1 fell after a bath, and the other fell from a height of about 2 metres. One patient had documented loss of consciousness. All of them had a Glasgow Coma Score (GCS) of 15/15 at the time of CT imaging. None of the cases subsequently revealed any evidence of traumatic brain injury on CTB but they all demonstrated mandibular condyle fractures best appreciated on coronal views. Axial (Fig. 1) and coronal (Fig. 2) images demonstrate a subtle fracture (arrows) of the mandible on the axial which is better appreciated on the coronal images.

In young children, symptoms of condylar fractures are subtle and may be overlooked. ${ }^{[4]}$ Clinical signs and symptoms described for mandibular fractures include haemorrhage from the external auditory meatus. ${ }^{[4]}$ One of the 4 patients had haemotympanum on clinical examination, and a СТВ was requested to exclude a base of skull fracture, but instead revealed a mandibular condyle fracture as the cause of the haemotympanum. Lacerations involving the chin are a commonly

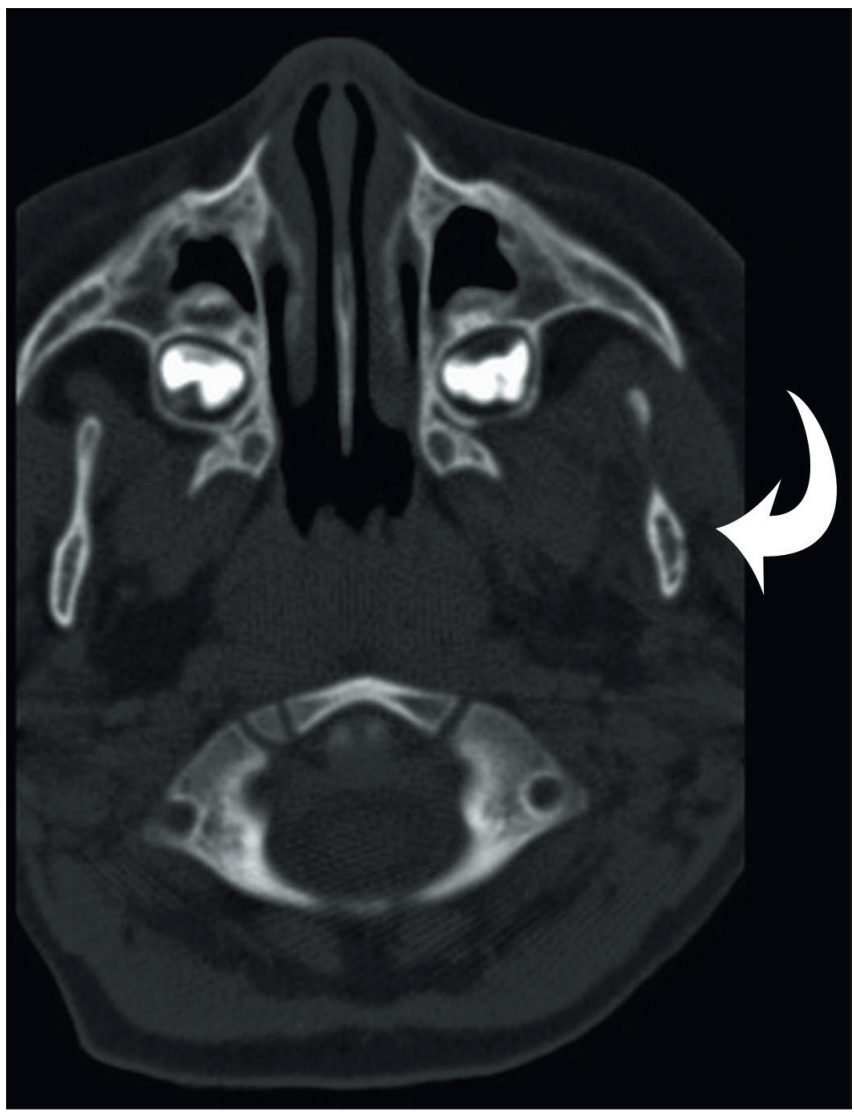

Fig. 1. Axial CT demonstrates a subtle left mandibular fracture (arrow). 


\section{RADIOLOGICAL TIPS}

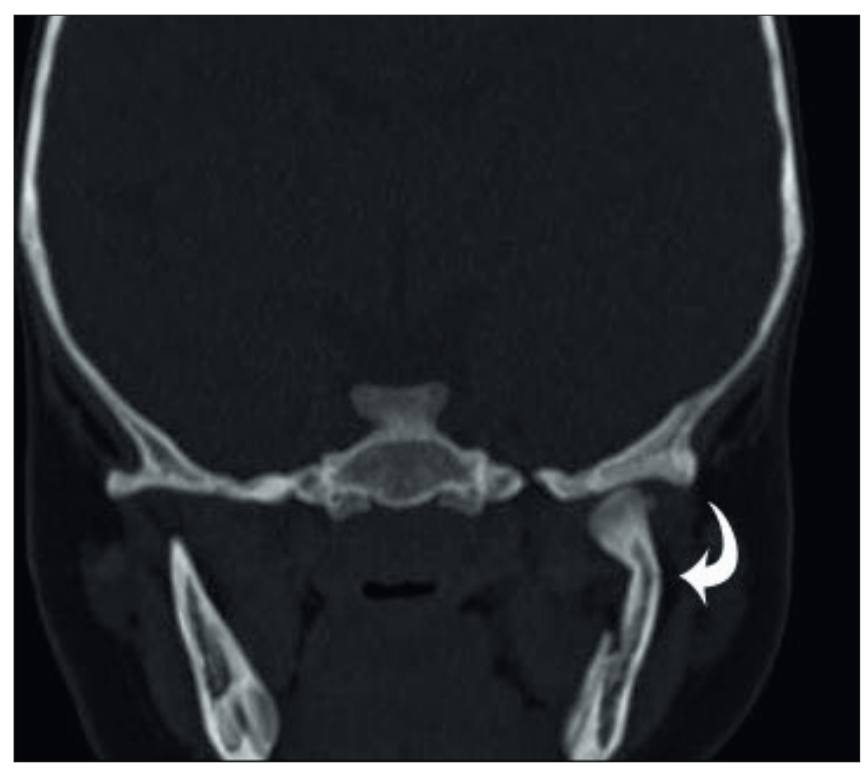

Fig. 2. The left mandibular fracture (arrowed) in the same patient is better appreciated in the coronal plane. associated clinical finding with mandibular fractures. ${ }^{[4]}$ Chin lacerations were documented in 2 of the 4 cases.

Overlooking mandibular fractures may have long-term consequences including facial asymmetry, malocclusion and ankylosis requiring invasive surgery for correction. ${ }^{[1,4]}$

Panoramic radiography is still recommended as first-line evaluation for mandibular fractures in children. ${ }^{[3]}$ CTB imaging often extends to involve the upper mandible/mandibular condyle in the imaging field. Routine review of the mandibular condyle particularly on the coronal views is advocated to avoid overlooking such fractures, particularly given the subtle clinical signs of mandibular fractures ${ }^{[4]}$ that may not always be appreciated or suspected by referring clinicians.

1. Alcala-Galiano A, Arribas-Garcia IJ, Martin-Perez MA, et al. Pediatric facial fractures: Children are not just small adults. Radiographics 2008;28:441-461.

2. Oslon RA, Fonseca RJ, Zeitler DL, et al. Fractures of the mandible: A review of 580 cases. J Oral Maxillofac Surg 1982;40:23-28.

3. Zimmerman CE, Troulis MJ, Kaban LB. Paediatric facial fractures: Recent advances in prevention, diagnosis and management. Int J Oral Maxillofac Surg 2006;35(1):2-13.

4. Myall RW, Sandor GK, Crystal EB. Are you overlooking fractures of the mandibular condyle. Pediatrics 1987;79(4):639-641. 